\title{
Realization of a Spatially Multiplexed MIMO System
}

\author{
David Samuelsson, Joakim Jaldén, Per Zetterberg, and Björn Ottersten \\ Department of Signals, Sensors, and Systems, Royal Institute of Technology (KTH), 10044 Stockholm, Sweden
}

Received 14 December 2004; Revised 1 April 2005; Accepted 5 April 2005

\begin{abstract}
Multi-antenna systems can provide improvements in wireless systems increasing spectral efficiency, reliability, range, and system capacity. Herein we show how some of the potentials of MIMO systems can be realized on a simple radio hardware platform by utilizing advanced real-time signal processing and coding. We present a real-time implementation of a 2 by 2 MIMO system employing spatial multiplexing to achieve high spectral efficiency in an indoor non-line-of-sight environment operating in the $1800 \mathrm{MHz}$ range. Well-known processing and coding techniques are employed and our contributions lie in: discussing implementational aspects and solutions often overlooked but critical for high-performance operation; demonstrating the degree to which the simple baseband AWGN model can be used to accurately model/predict the MIMO system on the current hardware; and demonstrating the feasibility of real-time spatial multiplexing achieving up to $15 \mathrm{bps} / \mathrm{Hz}$ on a 2 by 2 system in a realistic indoor environment with off-the-shelf radio hardware.
\end{abstract}

Copyright (c) 2006 David Samuelsson et al. This is an open access article distributed under the Creative Commons Attribution License, which permits unrestricted use, distribution, and reproduction in any medium, provided the original work is properly cited.

\section{INTRODUCTION}

Multiple antennas at the transmitter and receiver have emerged as one of the key technologies for increasing the spectral efficiency of future wireless communication systems. At this stage, many of the theoretical aspects of the multiple-antenna channel are well understood $[1,2]$. Similarly, a wealth of techniques exists for the exploitation of the potential gain offered by the multiple-antenna channel, both when the channel is assumed known to the transmitter and when it is not. Traditionally, space-time techniques have been designed either to maximize throughput or to increase robustness. However, recent theoretical results exist on the tradeoff between the two competing objectives [3]. Spatial multiplexing, which is employed in this project, is an example of a technique which aims at increasing the overall data rate. It does so by creating several virtual data paths across the wireless interface [4].

Most of these transmission techniques are based on simple linear discrete-time models of the multiple-antenna channel. While the signal processing required by many of these techniques is readily implemented in DSP software, it is essential that the model accurately reflects the underlying hardware as well as the actual wireless channel. Most common models of wireless channels implicitly assume accurate synchronization and carrier frequency offset compensation. It is thus important that such practical issues are dealt with in a fashion which makes them transparent to higher-level processing.

There has, to this date, been relatively few papers illustrating the feasibility of implementing multiple-antenna techniques on current hardware and evaluating the aforementioned models. A notable exception is the hardware implementation of the V-BLAST architecture $[5,6]$, where a spectral efficiency of $40 \mathrm{bps} / \mathrm{Hz}$ was achieved for a 12 by 8 MIMO system in laboratory settings. Other efforts in this area include the development and evaluation of MIMO OFDM systems in $[7,8]$.

In this paper, we demonstrate the feasibility of a multipleantenna technique by presenting a real-time implementation of a spatially multiplexed communication system operating over a 2 by 2 narrowband wireless multiple-input multipleoutput (MIMO) channel, in a non-line-of-sight indoor environment. The maximum supported data rate is adaptively obtained at the receiver and fed back to the transmitter. All in all, the system implemented is able to reach a spectral efficiency of up to $15 \mathrm{bps} / \mathrm{Hz}$.

The carrier frequency of the system is $1766.600 \mathrm{MHz}$ and the bandwidth of the channel is only $9.6 \mathrm{kHz}$. The system is mainly built with standard mini circuit components, but is equipped with a TI C6701 DSP in the transmitter, as well as receiver, module. This enables a substantial amount of real-time signal processing at both ends of the wireless link. The system is targeted towards a low mobility scenario where 


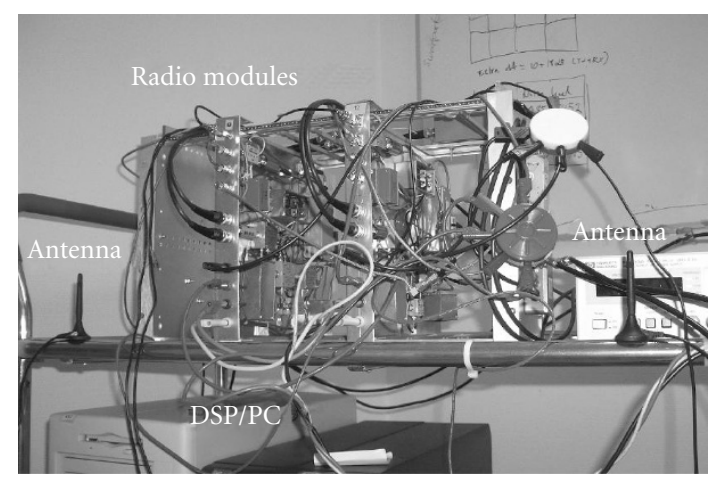

Figure 1: The figure shows the hardware setup of a two antenna receiver module.

TABLE 1: System summary.

\begin{tabular}{|c|c|}
\hline Antenna configuration & 2 by 2 MIMO \\
\hline Transmission scheme & Spatial multiplexing \\
\hline Modulation & $\begin{array}{l}\text { Trellis coded modulation. Adaptive bit } \\
\text { loading ranging from } 8 \text { PSK to } 512- \\
\text { cross QAM ( } 8 \text { cosets })\end{array}$ \\
\hline TCM code & $\begin{array}{l}\text { A } 2 / 3 \text {-rate, } 64 \text { state convolution trellis } \\
\text { code }\end{array}$ \\
\hline $\begin{array}{l}\text { Peak throughput } \\
\text { (goodput) }\end{array}$ & $2 \times 8$ bits/symbol \\
\hline Peak spectral efficiency & $15.6 \mathrm{bps} / \mathrm{Hz}$ \\
\hline Frame length & 32 symbols, $3.3 \mathrm{~ms}$ \\
\hline Super frame & 67 frames \\
\hline Feedback & $\begin{array}{l}\text { Wired feedback link (rate: } 32 \text { bits/ } \\
\text { frame) }\end{array}$ \\
\hline Feedback delay & 5 frames \\
\hline Carrier frequency & $1766.600 \mathrm{MHz}$ \\
\hline $\begin{array}{l}\text { Signaling rate } \\
\text { (bandwidth) }\end{array}$ & $9.6 \mathrm{kHz}$ \\
\hline Analog filter bandwidth & $14 \mathrm{kHz}$ \\
\hline Pulse shape filter & Root-raised cosine (roll-off $\beta=0.5$ ) \\
\hline Output power & $0.2 \mathrm{~mW}$ \\
\hline Receiver nose figure & approx. $10 \mathrm{~dB}$ \\
\hline Sampling & $48.0 \mathrm{kHz}$ (upsample factor of 5) \\
\hline DSP & TI C6701 \\
\hline
\end{tabular}

channel state information (CSI) can be accurately obtained and fed back to the transmitter. For a thorough description of the receiver and transmitter hardware see [9]. A photo of the hardware setup is given in Figure 1 and major system parameters are listed in Table 1.

In order to further illustrate the potential benefits of multiple antennas, the implemented system is compared to a single-antenna (SISO) system using the same hardware; implemented with identical temporal equalization and coding. By rapidly alternating between the MIMO and SISO implementations, the two systems can be compared on similar channel realizations. The 2 by 2 MIMO system is observed to have a substantial gain in throughput over the SISO system.
The throughput is typically increased by a factor of 2 for the MIMO system.

Although the wireless channel is close to frequency flat, a significant amount of intersymbol interference (ISI) is introduced by the nonideal characteristics of the radio hardware in the transmitter and receiver chains. Therefore, another contribution of this work is to effectively show that such imperfections, due to off-the-shelf radio hardware, can to a large extent be adaptively compensated for in software. To increase the resulting signal to interference and noise ratio (SINR), a linear temporal equalizer is applied on each of the received signals. An efficient implementation allows a jointly SINR maximizing channel and equalizer estimate to be computed and adaptively updated in real time.

Further, it is investigated under what conditions low-level functionality (such as synchronization and mitigation of the above mentioned ISI) may be separated from higher-level processing. This is done by evaluating when the simple memoryless discrete time linear model is an appropriate abstraction of the underlying hardware. The benefit of such separation of functionality is twofold. Firstly, it will generally allow significant reduction of complexity in the receiver. Secondly, it demonstrates that simple models often used in the literature are sufficient to accurately predict the performance of the proposed communication techniques within certain signalto-noise ratio (SNR) limits.

\subsection{System design strategy}

As indicated earlier, the system is designed in a layered structure. Essentially, the objective of each layer is to provide the layer above with an effective channel, where the inherent design assumptions of that layer are valid. This design approach is often adopted in communication systems. In the design, three different layers 1 ("coding layer," "spatial layer," and "temporal layer") are adopted.

The objective of the lowest layer, the temporal layer, is to provide the upper layers with a discrete-time channel which is well described by the memoryless linear MIMO channel given by

$$
\mathbf{y}(n)=\mathbf{H x}(n)+\mathbf{w}(n)
$$

Here $\mathbf{H} \in \mathbb{C}^{2 \times 2}$ is the effective channel matrix, $\mathbf{w}(n)$ is spatially and temporally white Gaussian noise, and $\mathbf{x}(n)$ are the symbols to be transmitted. This is illustrated by Figure 2. The properties of this channel are well known. This simplifies implementation and analysis, which motivates the layered design approach. Note that although we have chosen to implement a spatially multiplexed system, the structure of this temporal layer would be virtually unchanged if another technique was chosen. The details of the temporal layer are further described in Section 2.

\footnotetext{
${ }^{1}$ These layers should not be confused with the layers of the OSI network model. All the layers referred to here are components of the physical layer (layer 1) of the OSI model.
} 


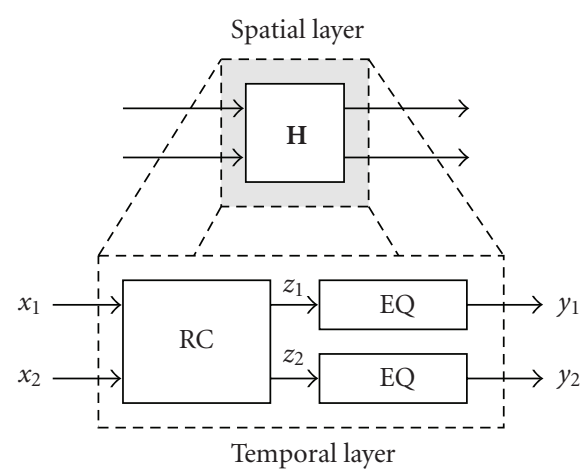

FIGURE 2: Illustration of the temporal and spatial design layers. The effective radio channel (RC), as seen from the transmitted symbols (digital base band) to the received signals, is shown as a single block in the temporal layer. By the use of digital temporal equalizers (EQ) the ISI introduced by hardware is mitigated and yields the flat fading MIMO channel model with gains given by $\mathbf{H}$.

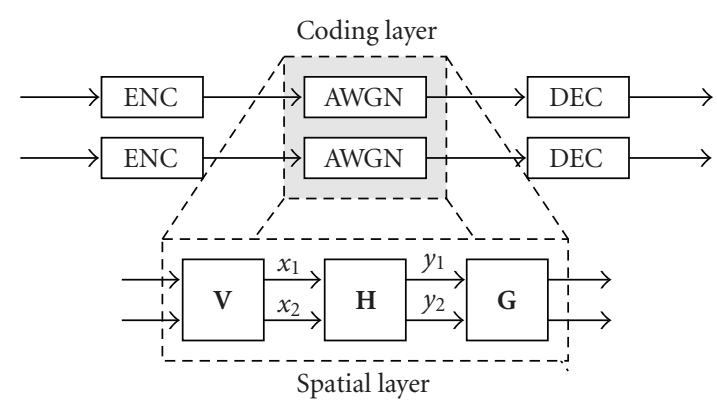

FIGURE 3: Illustration of spatial and code design layers. By the use of linear pre- and postprocessing, the spatial channel $\mathbf{H}$ is decoupled into two parallel AWGN SISO channels over which data is transmitted using a TCM scheme.

The next layer is characterized as a spatial layer. Given that the channel model (1) applies, two noninterfering spatial data paths are created. By linear pre- and postprocessing based on the singular value decomposition (SVD) of the channel matrix $\mathbf{H}$, two parallel additive white Gaussian noise (AWGN) channels are obtained. This is illustrated in Figure 3. In our implementation, the data on each such spatial channel is protected by trellis coded modulation (TCM) $[10,11]$. The structure of the pre- and postprocessing matrices are further described in Section 3.3.

\subsection{Notation}

The notations used in this paper are illustrated in Table 2.

\subsection{Outline}

The outline of this paper is as follows. First, in Section 2 the MIMO testbed on which the system is implemented is described. Next, in Section 3 an overview of the system design is given. Following the overview, additional details on
TABLE 2: Notations.

\begin{tabular}{ll}
\hline $\mathbf{X}$ & A matrix \\
$x_{i, j}$ & The $(i, j)$ th element of $\mathbf{X}$ \\
$\mathbf{x}_{j}$ & The $j$ th row of $\mathbf{X}$ \\
$\mathbf{X}$ & A column vector \\
$x_{j}$ & The $j$ th element of $\mathbf{x}$ \\
$\mathbb{C}$ & The complex field \\
$\{\cdot\}^{*}$ & Hermitian conjugate transpose \\
$\|\mathbf{x}\|$ & The Euclidean norm of the vector $\mathbf{x}$ \\
$\{\cdot\}^{\star}$ & Denotes an optimal point \\
$\arg \max _{\mathbf{x}} f(\mathbf{x})$ & Denote an $\mathbf{x}^{\star}$ which maximizes the real- \\
$\mathbf{X}_{\mathbf{L}}$ & valued function $f(\mathbf{x})$. \\
$\mathbf{x}_{\mathrm{L}}$ & The last 2 by 2 diagonal block of matrix $\mathbf{X}$ \\
$\sigma_{i}(\cdot)$ & The last 2 elements of vector $\mathbf{x}$ \\
\hline
\end{tabular}

the equalizer design are given in Section 4. In Section 5 several aspects of implementing a MIMO system on a hardware platform are discussed. These include: spatial and temporal equalizer implementation, feedback quantization, and protection against bit-errors on a fading channel.

Next, the performance of the system is evaluated. The validity of the memoryless discrete time AWGN channel model of (1) is assessed in Section 6. It is shown, by measurements, that under realistic operating conditions, the memoryless AWGN channel model accurately describes the effective channel. The benefits of MIMO systems are evaluated in Section 7. A thorough comparison to single-antenna performance is presented. This is accomplished by the implementation of a single-antenna system using identical temporal processing, hardware, and coding. It is shown that the multipleantenna system can achieve a throughput of up to, and above, twice that of the single-antenna system. Finally, this work is summarized in Section 8.

\section{THE MIMO TESTBED}

As mentioned above, the 2 by 2 MIMO testbed is built with radio equipment which introduces significant channel impairments in the form of ISI and frequency offsets. A key contribution is to demonstrate reliable, high-performance communication on such radio hardware. An overview of the system setup, including wireless interface and feedback link, is given in Figure 4.

\subsection{Hardware design}

In the following, the transmitter, as well as the receiver, is divided in two different stages. The first stage of the transmitter corresponds to base band preprocessing, which prepares the symbols to be transmitted over the two antennas. In the second stage, the transmission stage, pulse shaping (PS) is applied and the signals are D/A converted and up-converted to the carrier frequency. The two transmitter chains (one per antenna) of the transmission stage are illustrated and further 


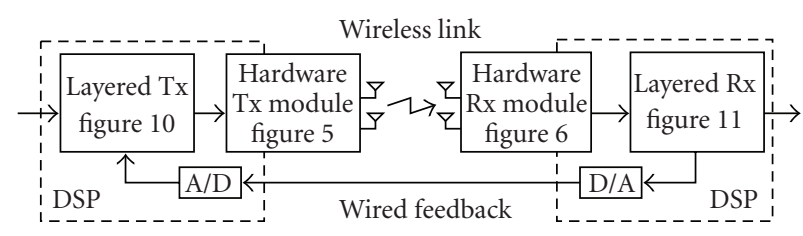

Figure 4: A system overview. The individual blocks of the system are further described in the indicated figures. The parts of the system physically located on the DSP cards are indicated by dashed lines.

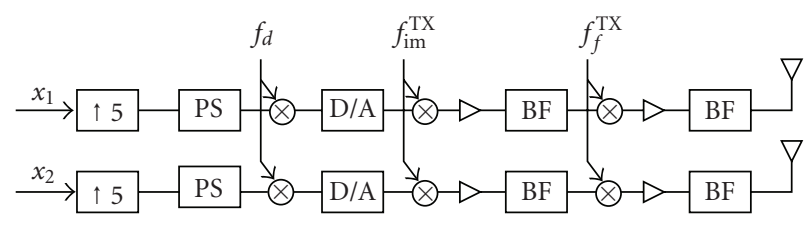

FIGURE 5: Illustration of the hardware transmitter modules. Before the baseband symbols $\mathbf{x}(n)$ are pulse shaped (PS), they are upsampled with a factor of 5 . After pulse shaping, they are digitally upconverted to center frequency $f_{d}=10 \mathrm{kHz}$ and D/A converted. The analog signals are further upconverted, amplified, and bandpass filtered (BF) in two stages before being transmitted with center frequency $f_{c}^{\text {TX }}$ on the two antennas.

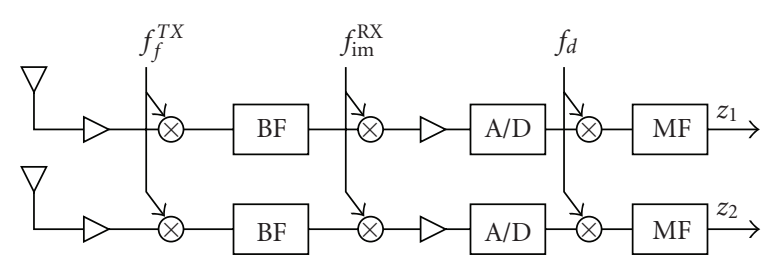

FIGURE 6: Illustration of the hardware receiver modules. The received signals are initially amplified, downconverted, and bandpass filtered. Before the signals are A/D converted, they are further downconverted and amplified. Finally the signals are digitally match filtered (MF), resulting in the upsampled received data streams, $\mathbf{z}(n)$.

described in Figure 5. For further details and list of components, see [9].

Similarly, the first stage of the receiver, the receiver stage, corresponds to amplification, down-conversion, and A/D conversion, as well as digital-matched filtering (MF) of the received signals. This is illustrated and further described in Figure 6; see also [9] for more details. The receiver stage supplies the second stage with the matched filtered signal, upsampled to 5 times the symbol rate, from which the transmitted symbols are to be decoded. The pulse shaping of the symbols is done digitally after up-sampling. A root raised cosine pulse is used for pulse shaping and for the corresponding matched filtering at the receiver.

The A/D and D/A conversions are performed with 16bit precision and with the relatively low sampling rate of
$48 \mathrm{kHz}$. The quantization level is thus high enough to disregard quantization noise, but the low sampling rate limits the system bandwidth, as well as the digital upconversion.

\subsection{System characteristics}

Throughout this work, the transmission and receiver stage are considered fixed and nontouchable, and artifacts of the imperfections introduced in the hardware are to be handled by the base band processing stages. This design view underlines the desire to compensate hardware imperfections with more flexible signal processing algorithms.

In Figure 7, a plot of a typical realization of the impulse response $p(t)$ of the entire transmitter-receiver chain (for a 1 by 1 system) is given. The impulse response has been normalized to make the zeroth tap real and positive with unit norm, that is, $p(0)=1$. This eliminates the complex base band equivalent gain between the two antennas.

Two important imperfections of the hardware may be observed in this figure. Firstly, the zero crossings of the impulse response do not coincide with the symbol positions, which results in substantial ISI when operating in the high SNR region. Secondly, the imaginary part of the impulse response causes a leakage between the in-phase (I) and quadrature (Q) signals.

The hardware induced ISI is caused by the bandpass filters and amplifiers, see Figures 5 and 6 . The filters have a $3 \mathrm{~dB}$ bandwidth of $14 \mathrm{kHz}$, which is not much higher than the symbol rate, in particular as frequency offsets of up to $2 \mathrm{kHz}$ may appear. Such a design relaxes the requirements on A/D converter sample rate and linearity as the filters rejects signals in adjacent frequency channels which may potentially be much stronger than the desired signal.

The frequencies of the oscillators are unlocked and therefore drift, and may be as much as $2 \mathrm{kHz}$ offset. A design which allows such impairments also contributes in decreasing costs. However, these frequency drifts change the impulse response of the channel, since different parts of the bandpass filters are utilized. The sample-phase of the A/D and $\mathrm{D} / \mathrm{A}$ converters is also variable up to half a sample, and is also slightly drifting. This also changes the effective channel. The above is the main motivation for adaptive equalization.

Before the signals are transmitted, they are bandpass filtered in two stages in order to limit the interference to neighboring frequency channels. The spectrum of the transmitted signal is given in Figure 8 . The $3 \mathrm{~dB}$ bandwidth of the modulated data is approximately $9600 \mathrm{kHz}$. Note also the side peak which is due to leakage of the intermediate oscillator $f_{\mathrm{im}}^{\mathrm{TX}}$ in Figure 5 . This peak falls only $10 \mathrm{kHz}$ from the desired signals due to the low first frequency $f_{d}$. If this frequency had been selected higher, the oscillator signal would have been completely eliminated in the band-pass filter. However, this was not possible due to the antialias filter integrated with the D/A converter which limits the output frequency to $20 \mathrm{kHz}$ (the D/A converter is intended for audio applications). It should also be mentioned that this, out of band, signal is not used at the receiver in any way, but rather strongly attenuated by the bandpass filters at the receiver. 


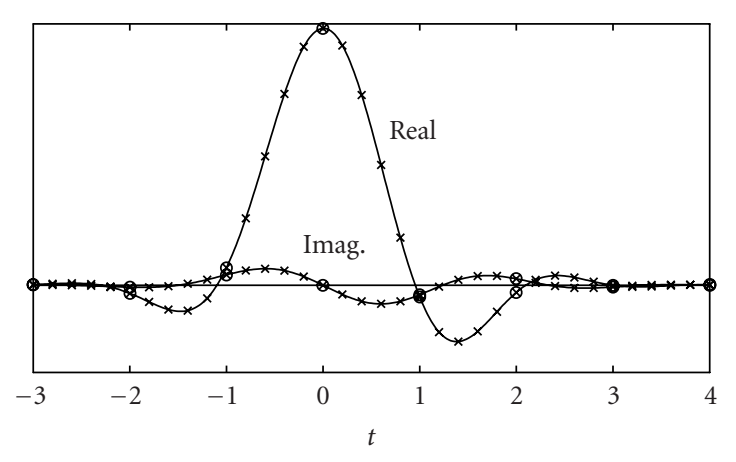

$\times$ Sample position

- Symbol position

Figure 7: The temporal impulse response $p(t)$ of the transmitterreceiver chain.

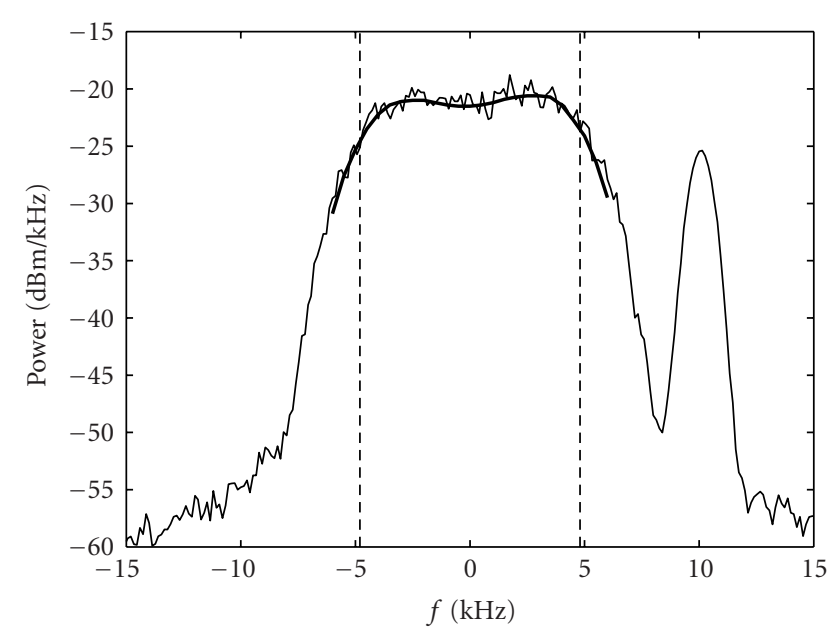

FIgURE 8: Illustrates the spectrum of the transmitted signal, centered at the carrier frequency. The spectrum was measured using a Rhode Schwartz FSH3 spectrum analyzer. Superimposed is a higher accuracy measurement using transmission of individual sinusoids. The dashed line indicates the $9600 \mathrm{kHz}$ bandwidth. Also note the side peak, which is an artifact of the intermediate upconversion frequency, $f_{\mathrm{im}}^{\mathrm{TX}}$.

\subsection{Feedback link}

In order to utilize channel state information a (wired) feedback link from the receiver to the transmitter is provided. This feedback link allows a 32-bit word to be fed back to the transmitter for each frame of 32 symbol pairs that is received. Inherent in the system design is a feedback delay of five frames and therefore the feedback from the first frame is not available for processing until the sixth frame.

\section{SYSTEM OVERVIEW}

In this section the base band processing of the implemented system is described. The processing can be divided into three

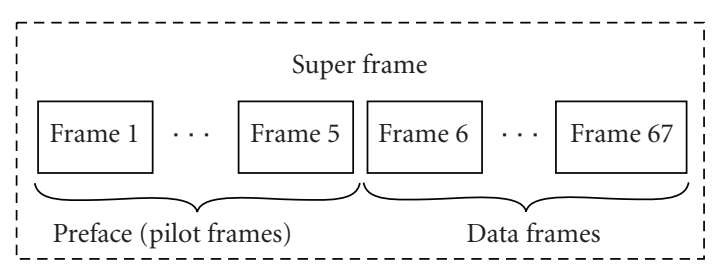

Figure 9: Illustration of the frame structure. The first 5 frames are devoted to pilot signaling, whereas each of the remaining 62 frames contain a block of TCM encoded data symbols. A group of 67 frames constitute a super frame.

main categories:

(1) coding and detection (coding layer),

(2) spatial multiplexing (spatial layer),

(3) temporal processing (temporal layer).

There is a wealth of literature describing each of these categories, most of which assumes that the channel may be well approximated by some simple mathematical model. When designing each of these categories it is thus essential that each part is supplied with the appropriate level of abstraction in which the inherent assumptions are valid.

It should be mentioned that we do not claim this layered design to be optimal from a performance perspective, but rather a convenient design approach which enables the use of well-known coding and spatial processing techniques. However, as shown in Section 6, for a wide range of SNRs, the performance penalty is relatively small.

\subsection{Frame structure}

The system operates on a frame by frame basis. Each frame has 32 symbol pairs, one for each stream, and is coded and decoded separately. The first 5 frames, the preface, are devoted to pilot signaling for synchronization and frequency offset, channel and equalizer estimation. By the sixth frame, the feedback, processed from the first frame, is available at the transmitter and the next 62 frames are used for data transmission. In total, 67 frames are transmitted, which constitute a super frame. The frame structure is illustrated in Figure 9.

\subsection{Coding and detection}

In order to protect the data and to maintain a low bit error rate (BER), coding with adaptive data rate is implemented. Since the wireless channel is of slow fading nature, adaptive rate TCM $[10,11]$ is well suited for the application. It is however not straightforward to implement such a code on the MIMO channel with memory introduced by the ISI. Therefore, when implementing the coding, lower level layers should provide an effective channel, which is well approximated by two memoryless parallel AWGN channels, with a coherence time much larger than the duration of a frame.

Each of these parallel AWGN channels is encoded separately by a 64 state TCM code. For simplicity, the power is 
equally distributed over the two streams. The BER is kept below a threshold by adapting the data rate by choosing an appropriate constellation size ranging from 8 PSK up to 512 cross QAM. This corresponds to data rates ranging from $17.4 \mathrm{kbps}$ up to $75 \mathrm{kbps}$ for each spatial channel. The peak throughput of the system is $150 \mathrm{kbps}$, corresponding to a spectral efficiency of more than $15 \mathrm{bps} / \mathrm{Hz}$. Note that these rates refer to the information bits and disregards coding redundancy. The TCM code is discussed in more detail in Appendix A.

It should be mentioned that encoding each stream separately is a suboptimal approach. That is, from a performance perspective it is preferable to use a single code over both spatial streams, as well as to distribute power according to the water filling principle. However, in the high SNR region, equal power allocation is close to optimal. The performance penalty of the suboptimal approach is also well motivated by implementational simplicity.

\subsection{Spatial multiplexing}

The MIMO channel is characterized by cross talk between the transmitted signals, contrary to the noninterfering parallel channel assumption made when designing the coding. Spatial multiplexing is a technique which exploits the spatial dimensions exclusively to maximize the throughput rather than utilizing the potential diversity increase of the MIMO channel [3]. This is accomplished by creating two parallel (noninterfering) spatial channels, as expected by the coding layer.

Spatial multiplexing is applicable whenever it is reasonable to assume that the transmitter, as well as the receiver, has accurate CSI. In the current indoor, low mobility scenario, where the channel fades very slowly, the relevant CSI can be fed back from the receiver to the transmitter.

For the spatial multiplexing, it is assumed that the wireless system is well approximated by the linear memoryless discrete time channel model (1). By appropriate processing in the temporal layer, this is a valid assumption.

By pre-multiplying the symbols to be transmitted, $\mathbf{c}$, by $\mathbf{V}$ and the received symbols by $\mathbf{G}=\boldsymbol{\Sigma}^{-1} \mathbf{U}^{*}$, where $\mathbf{H}=\mathbf{U} \boldsymbol{\Sigma} \mathbf{V}^{*}$ is the SVD of the channel matrix, the MIMO channel can be decoupled into two parallel channels [4]:

$$
\widehat{\mathbf{c}}=\mathbf{G y}=\mathbf{G}(\mathbf{H x}+\mathbf{w})=\mathbf{G H V c}+\mathbf{G w}=\mathbf{c}+\tilde{\mathbf{w}} .
$$

Here $\tilde{\mathbf{w}}$ is spatially white complex Gaussian noise with covariance matrix $N_{0} \boldsymbol{\Sigma}^{-2}$.

The two parallel channels resulting from the spatial multiplexing are well suited for the assumptions made in the coding layer.

\subsection{Temporal processing}

The temporal layer should provide the spatial layer with the right level of abstraction. That is, the system should be well approximated by a memoryless linear channel model (1) which operates on a symbol by symbol basis rather than the upsampled data provided by the matched filtered signals.
The most obvious temporal processing is the temporal equalizer, which efficiently removes most of the ISI. The temporal equalizer thus ensures that the channel is well approximated as memoryless. This corresponds to providing the spatial layer with a channel with small ISI, that is, high SINR.

There are however many other key issues that must be handled before the spatial processing is applicable. These include synchronization and carrier frequency offset compensation.

\subsubsection{ISI mitigation}

In the implementation a complex valued 9 tap temporal equalizer is implemented at the receiver for each antenna, see Figure 2 . The equalizer taps are initially estimated jointly with the channel matrix using pilot signals. The equalizer and channel matrix estimates are then adaptively updated throughout the super frame using decision directed feedback of the decoded data. The estimates are chosen to jointly maximize the SINR of the resulting link, see Section 4.2. This joint estimation of the channel and equalizer is made possible by an efficient implementation, see Section 5.1, where an optimization algorithm is presented. The algorithm complexity is dominated by a Cholesky factorization of an $11 \times 11$ matrix.

Note that it is not possible to remove all the ISI at the receiver only by equalizing the two data streams separately. However the performance loss is small, unless the system is operating at very high SNRs, see Section 6.

The choice of number of taps in the equalizer is a tradeoff between performance and computational complexity. It was observed that increasing the number of equalizer taps beyond 9 only has a marginal effect on the performance, and the increased computational complexity is not motivated. It should further be noted that when the number of equalizer taps are restricted, a nonuniform spacing of the taps is preferable. This is further discussed in Section 5.1.3.

The statistical properties of the residual noise is further analyzed in Section 6. It is concluded that the residual noise, is well approximated as independent white Gaussian, indicating that the equalizer is operating as designed.

\subsubsection{Synchronization and frequency offset compensation}

The frequency offset compensation is the first processing performed by the receiver. Each sample of the received signals is phase rotated to compensate for the frequency mismatch. In subsequent processing the affect thereof may be disregarded.

The carrier frequency offset is estimated prior to any other signal processing at the receiver, such as synchronization and channel estimation. The frequency offset must therefore initially be estimated, from the first received pilot frame, without synchronization and knowledge of the channel. These obstacles are overcome by the use of a pilot frame with repetitive structure [12]. This allows the frequency offset to be estimated from the autocorrelation of the received signal. The technique described in [12] is readily extended to the MIMO case, exploiting that the offset can be assumed 


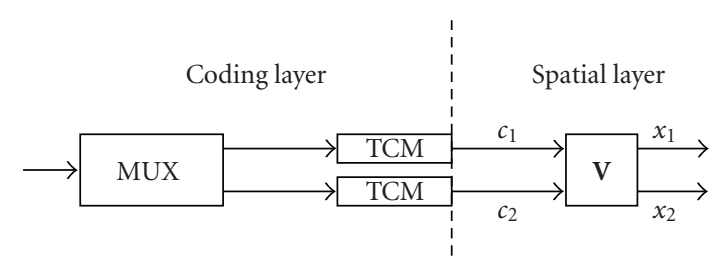

FIGURE 10: Illustration of the transmitter structure. The binary input data stream is split into two separate streams, one for each spatial channel. The bit stream for each spatial channel is TCM encoded into 32 symbols, $c_{j}(t)$ for $t=1, \ldots, 32$, which makes up one frame. Before the symbols are transmitted, they are multiplied with the spatial multiplexing preprocessing matrix $\mathbf{V}$.

to be the same on the transmitting and receiving antenna pairs. The accuracy of the frequency offset estimate is improved by updating the estimate throughout the entire preface of the super frame. The pilot frame is transmitted repeatedly throughout the preface, and by correlating the received buffer of the current frame with that of the first, a highly accurate estimate is acquired.

After frequency offset estimation and correction, synchronization is performed. Due to the adaptive nature of the equalizer, the system is not sensitive to small synchronization errors, but a rough synchronization must initially be performed to locate the frame. This is done by cross-correlating the match-filtered received buffer with the pilot frame.

\subsection{Transmitter and receiver structure}

The above discussion has focused on the different layers of abstraction required for the design of the system. This section summarizes the above by illustrating how the actual transmitter and receiver are designed. The transmitter and receiver structures are described in Figures 10 and 11, respectively. The different processing layers are marked in the figures. Note that the temporal layer does not affect the transmitter in this implementation.

When the spatial filter has been applied at the receiver, it is assumed that the channel as seen from $c_{j}$ to $\hat{c}_{j}$ can be well approximated by the AWGN channel assumed in the TCM code design.

The transmitter adapts the bit rate on the two spatial streams to match the quality of the two spatial channels. The receiver estimates the SNRs on the spatial channels and feeds back the maximum supported rate. The rate is modified by changing the constellation size used by the TCM code, see Appendix A.

Further, the spatial preprocessing matrix is computed at the receiver and fed back to the transmitter.

\section{EQUALIZER DESIGN}

In this section a more detailed description of the temporal equalizer is given. The use of separate equalizers for the two

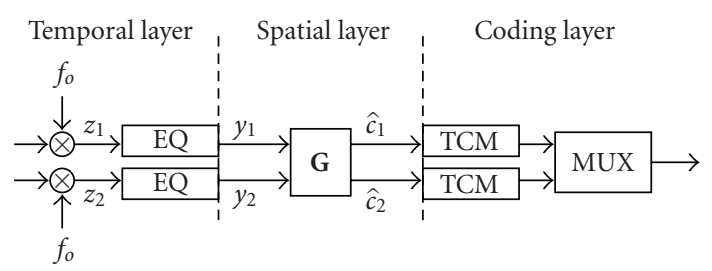

FIGURE 11: Illustration of the receiver structure. After the initial synchronization, the received up-sampled signals are phase rotated to compensate for the carrier frequency offset. Next the ISI is suppressed by applying temporal equalizers. The two spatial streams are then separated by the linear decorrelating spatial filter $\mathbf{G} \in$ $\mathbb{C}^{2 \times 2}$. The two streams are finally maximum-likelihood decoded and merged to a single-bit streams.

antennas are first motivated. Next, the optimization problem yielding a jointly SINR maximizing channel and equalizer is formulated.

\subsection{Design assumptions}

Assuming stationary conditions and a frequency flat wireless channel, the signal $z_{1}$ may be expressed as (ignoring noise)

$$
z_{1}(k)=\sum_{n} \tilde{h}_{11} p_{11}(k-5 n) x_{1}(n)+\tilde{h}_{12} p_{12}(k-5 n) x_{2}(n),
$$

where $p_{11}$ and $p_{12}$ are time discrete descriptions of the temporal symbol spreading experienced from $x_{1}$ and $x_{2}$ to $z_{1}$, respectively, and where $\widetilde{h}_{i j}$ is the baseband equivalent complex gain form antenna $j$ to $i$. Note that the upsampling factor of 5 is included in the above. Essentially, $p_{11}$ and $p_{12}$ are impulse responses such as depicted in Figure 7. Under the additional assumption that the transmitter chains, that is, the signal path form $x_{1}$ and $x_{2}$ to respective antenna, are identical, the expression further simplifies to

$$
z_{1}(k)=\sum_{n} p_{1}(k-5 n)\left[\tilde{h}_{11} x_{1}(n)+\tilde{h}_{12} x_{2}(n)\right],
$$

where now $p_{1}=p_{11}=p_{12}$. Thus, under the assumption that the transmitter chains are equal and that the wireless channel is frequency flat, the spatial and temporal self interference may be decoupled and described separately. This motivates an approach where first the temporal ISI is suppressed to yield a frequency flat channel, where only the spatial interference remains. In the present scenario where temporal ISI is introduced by hardware imperfections and not by the wireless channel itself, it seems reasonable to assume that this ISI could be suppressed by processing the data streams sampled in each of the receiver chains separately. Further, such processing may be located in the receiver alone. This is indeed the approach indicated in Figure 2.

The validity of this approach depends on the applicability of the assumptions on which it is based. The assumption that the two transmitter chains are identical is admittedly questionable due to the imprecise nature of the hardware. There are however several reasons to still make this assumption. 
Firstly, accounting for differences in the transmitter chains requires processing at the transmitter as well as receiver. Due to the time varying nature of the ISI patterns, this involves continuously estimating the impulse response and feeding it back to the transmitter. Such an approach severely complicates the system design due to increasing demands on the feedback link, especially when the number of taps in the equalizer is large.

There are, however, also physical reasons which motivate such an assumption. One of the main reasons for the time variations of the ISI is that the mixing frequencies change slowly over time. As explained in Section 2, the signal will drift in frequency relative to the pass bands of the bandpass filters. This will cause a change in the overall impulse response. Since the same oscillator supplies the mixing frequency for both transmitter chains, this effect is similar in both chains. Therefore, the assumption that the transmitter chains are identical is reasonable as long as the difference in bandpass filters and amplifiers may be considered small in comparison to the noise level, even in the presence of large scale time variations.

\subsection{Channel and equalizer estimation}

The equalized signal $\mathbf{Y}$ is given by

$$
\mathbf{Y}=\left[\begin{array}{l}
\mathbf{y}_{1} \\
\mathbf{y}_{2}
\end{array}\right] \in \mathbb{C}^{2 \times 32},
$$

where the elements of $\mathbf{y}_{i} \in \mathbb{C}^{1 \times 32}$ are the symbols associated with the signal received on antenna $i$ during the frame. This equalized signal is obtained as

$$
\mathbf{y}_{i}=\mathbf{d}_{i} \widetilde{\mathbf{Z}}_{i}
$$

where the elements of $\mathbf{d}_{i} \in \mathbb{C}^{1 \times 9}$ are the equalizer taps of the $i$ th equalizer, and the elements of $\widetilde{\mathbf{Z}}_{i} \in \mathbb{C}^{9 \times 32}$ are the elements of the matched filtered received buffer arranged appropriately. The $j$ th column of $\widetilde{\mathbf{Z}}_{i}$ consists of the samples on which the equalizer should operate to acquire the $j$ th received symbol on antenna $i$. Note also that $\widetilde{\mathbf{Z}}_{i}$ depends on the synchronization.

The main objective of the equalizer is to make the equalized symbols well approximated by the linear channel model

$$
\mathbf{Y}=\mathbf{H X}+\mathbf{W}
$$

where $\mathbf{H} \in \mathbb{C}^{2 \times 2}$ is a channel matrix, $\mathbf{X} \in \mathbb{C}^{2 \times 32}$ contains the transmitted symbols, and the elements of $\mathbf{W}$ are independent complex Gaussian noise. In our case, the residual $\mathbf{W}$ is a mixture of noise and ISI. The noise and ISI of the received stream $i$ is thus given by the residual $\mathbf{w}_{i}=\mathbf{d}_{i} \widetilde{\mathbf{Z}}_{i}-\mathbf{h}_{i} \mathbf{X}$, where $\mathbf{w}_{i}$ and $\mathbf{h}_{i}$ are the $i$ th row of $\mathbf{W}$ and $\mathbf{H}$, respectively.

The equalizer of stream $i$ is based on (7) and is designed to maximize the SINR of the equalized streams:

$$
\operatorname{SINR}_{i}=\frac{\left\|\mathbf{h}_{i} \mathbf{X}\right\|^{2}}{\left\|\mathbf{w}_{i}\right\|^{2}}=\frac{\left\|\mathbf{h}_{i} \mathbf{X}\right\|^{2}}{\left\|\mathbf{d}_{i} \widetilde{\mathbf{Z}}_{i}-\mathbf{h}_{i} \mathbf{X}\right\|^{2}} .
$$

This suggests joint channel and equalizer estimation, which is performed in the temporal layer. The estimated channel is however utilized within the spatial layer.

The jointly optimal channel and equalizer estimates are thus obtained as

$$
\left(\mathbf{d}_{i}^{\star}, \mathbf{h}_{i}^{\star}\right)=\underset{\mathbf{d}_{i}, \mathbf{h}_{i}}{\arg \max } \frac{\left\|\mathbf{h}_{i} \mathbf{X}\right\|^{2}}{\left\|\mathbf{d}_{i} \widetilde{\mathbf{Z}}_{i}-\mathbf{h}_{i} \mathbf{X}\right\|^{2}},
$$

which is solved in [13], where the problem arises in the similar context of joint space-time processing. A computationally efficient algorithm was also given, for the special case when the transmitted data is an orthogonal pilot sequence, that is, $\mathbf{X X}^{*}$ is a scaled identity matrix. In the current scenario, this is not the case.

Herein a mathematically different approach is used to derive an efficient algorithm, with close to identical computational complexity, but where no assumptions on the transmitted data are required. The most computationally complex step in this algorithm is the Cholesky factorization of an 11 by 11 matrix. The algorithmic solution of (9) is further discussed in Section 5.1.

\section{ASPECTS OF SYSTEM IMPLEMENTATION}

This section provides a more in-depth description of some key implementation issues. Factors such as feedback delay and feedback quantization and how these affect the spatial equalizer are discussed. The temporal equalizers are also described thoroughly. In addition to the derivation of a computationally efficient algorithm for joint equalizer and channel estimation, aspects such as spacing of the equalizer taps are discussed.

The purpose of this section is to provide the reader with details regarding implementation and may be skipped by the reader only interested in an overview, as subsequent sections do not rely on its content.

\subsection{Temporal equalizer}

Below the implementation of the joint channel matrix and equalizer tap estimation is discussed. It is shown that the SINR maximizing channel and equalizer is the solution to an 11 dimensional generalized eigenvalue problem. It is further explored how the complexity of this optimization can be dramatically reduced to an ordinary 2 dimensional eigenvalue problem, by appropriate variable substitutions.

As previously mentioned, the optimization problem in (9) was previously solved in [13]. A low complexity algorithm was also derived, but required a special orthogonal structure on the data streams, which is not applicable in our context. The algorithm derived below, using a different mathematical approach, preserves the low complexity without any such assumptions.

In Algorithm 1, the joint channel and equalizer estimation algorithm is summarized. Below a thorough description of each step of the algorithm is given. 

(1) $\mathbf{S} \leftarrow \mathbf{X X}^{*} \in \mathbb{C}^{2 \times 2}, \mathbf{P} \leftarrow \mathbf{X} \tilde{\mathbf{Z}}^{*} \in \mathbb{C}^{2 \times 9}$, $\mathbf{Q} \leftarrow\left(\tilde{\mathbf{Z}} \tilde{\mathbf{Z}}^{*}+\delta \mathbf{I}\right) \in \mathbb{C}^{9 \times 9}$
(2) $\mathbf{A} \leftarrow\left[\begin{array}{cc}\mathbf{Q} & -\mathbf{P}^{*} \\ -\mathbf{P} & \mathbf{S}\end{array}\right]$
(3) Cholesky factorize $\mathbf{A}$ into $\mathbf{R}^{*} \mathbf{R}$
(4) $\widetilde{\mathbf{B}}_{\mathrm{L}} \leftarrow \mathbf{R}_{\mathrm{L}}^{-*} \mathbf{S R}_{\mathrm{L}}^{-1}$
(5) Solve $\widetilde{\mathbf{B}}_{\mathrm{L}} \mathbf{q}_{\mathrm{L}}=\lambda_{\max } \mathbf{q}_{\mathrm{L}}$
(6) Solve $\mathbf{R} \mathbf{p}=\left[\mathbf{0}, \mathbf{q}_{\mathrm{L}}^{*}\right]^{*}$, using back substitution.
(7) $\mathbf{p}^{\star} \leftarrow \sqrt{32}\left|p_{1}\right| /\left(p_{1} \sqrt{\left\|\mathbf{q}_{\mathrm{L}}\right\|^{2}-\delta\|\mathbf{d}\|^{2}}\right) \mathbf{p}$

(8) Optimal equalizer $\mathbf{d}$ and channel $\mathbf{h}$ are obtained as $\left[\mathbf{d}^{\star}, \mathbf{h}^{\star}\right]=\left(\mathbf{p}^{\star}\right)^{*}$

Algorithm 1: Algorithmic solution to the joint SINR maximizing channel and equalizer design.

\subsubsection{The optimization algorithm}

In order to analyze the optimization problem (9), it is helpful to rewrite the SINR as a quadratic form:

$$
\operatorname{SINR}_{i}=\frac{\left[\mathbf{d}_{i}, \mathbf{h}_{i}\right]\left[\begin{array}{cc}
\mathbf{0} & \mathbf{0} \\
\mathbf{0} & \mathbf{X X}
\end{array}\right]\left[\mathbf{d}_{i}, \mathbf{h}_{i}\right]^{*}}{\left[\mathbf{d}_{i}, \mathbf{h}_{i}\right]\left[\begin{array}{cc}
\widetilde{\mathbf{Z}}_{i} \widetilde{\mathbf{Z}}_{i}^{*} & -\widetilde{\mathbf{Z}}_{i} \mathbf{X}^{*} \\
-\widetilde{X}_{i}^{*} & \mathbf{X X}
\end{array}\right]\left[\mathbf{d}_{i}, \mathbf{h}_{i}\right]^{*}} \triangleq \frac{\mathbf{p}_{i}^{*} \mathbf{B} \mathbf{p}_{i}}{\mathbf{p}_{i}^{*} \mathbf{A}_{i} \mathbf{p}_{i}}
$$

where $\mathbf{p}_{i}=\left[\mathbf{d}_{i}, \mathbf{h}_{i}\right]^{*}$ represents both the channel and the equalizer taps. $\mathbf{A}_{i} \in \mathbb{C}^{11 \times 11}$ is a positive definite (PD) matrix and $\mathbf{B} \in \mathbb{C}^{11 \times 11}$ is a sparse positive semidefinite (PSD) matrix. Note that only the right lower 2 by 2 block of $\mathbf{B}$ is nonzero. The sparse structure will be exploited fully in the implementation described below. Since the implementations of the two equalizers are identical, the index denoting the corresponding antenna is dropped in the following discussion.

From (10), it follows that the SINR is limited above by

$$
\operatorname{SINR} \leq \lambda_{\max }(\mathbf{B}, \mathbf{A}),
$$

where $\lambda_{\max }(\mathbf{B}, \mathbf{A})$ denotes the largest generalized eigenvalue of the eigensystem $\mathbf{B p}=\lambda \mathbf{A p}$. Equality is attained for the associated generalized eigenvector $\mathbf{p}^{\star}$, which is the optimal solution to the joint channel and equalizer estimator (9).

The sparse structure of $\mathbf{B}$ does however allow an efficient computation of $\mathbf{p}^{\star}$. By factoring $\mathbf{A}=\mathbf{R}^{*} \mathbf{R}$ using the Cholesky factorization into the upper triangular $\mathbf{R}$, the SINR may be written as

$$
\operatorname{SINR}=\frac{\mathbf{p}^{*} \mathbf{B} \mathbf{p}}{\mathbf{p}^{*} \mathbf{R}^{*} \mathbf{R} \mathbf{p}}=\frac{\mathbf{q}^{*} \mathbf{R}^{-*} \mathbf{B} \mathbf{R}^{-1} \mathbf{q}}{\mathbf{q}^{*} \mathbf{q}}=\frac{\mathbf{q}^{*} \widetilde{\mathbf{B}} \mathbf{q}}{\|\mathbf{q}\|^{2}},
$$

where $\mathbf{q} \triangleq \mathbf{R} \mathbf{p}$. The optimal $\mathbf{q}^{\star}$ which maximize the SINR is given by the eigenvector of $\widetilde{\mathbf{B}} \triangleq \mathbf{R}^{-*} \mathbf{B} \mathbf{R}^{-1}$ corresponding to the largest eigenvalue. The generalized eigenvalue problem has thus been reduced to an ordinary eigenvalue problem.

What is of more interest is that the special sparse structure of $\mathbf{B}$ is preserved in $\widetilde{\mathbf{B}}$. This follows since the quadratic form in (10) was designed such that only the lower right 2 by 2 block is nonzero and that $\mathbf{R}^{-*}$ and $\mathbf{R}^{-1}$ are lower and upper triangular, respectively. Hence $\widetilde{\mathbf{B}}$ is given by

$$
\widetilde{\mathbf{B}}=\left[\begin{array}{cc}
\mathbf{0} & \mathbf{0} \\
\mathbf{0} & \mathbf{R}_{\mathrm{L}}^{-*} \mathbf{X X}^{*} \mathbf{R}_{\mathrm{L}}^{-1}
\end{array}\right]=\left[\begin{array}{cc}
\mathbf{0} & \mathbf{0} \\
\mathbf{0} & \widetilde{\mathbf{B}}_{\mathrm{L}}
\end{array}\right],
$$

where subscript $\{\cdot\}_{\mathrm{L}}$ denotes the bottom right 2 by 2 block of a matrix. In the above, $\left(\mathbf{R}^{-1}\right)_{\mathrm{L}}=\mathbf{R}_{\mathrm{L}}^{-1}$ was used (since $\mathbf{R}$ is triangular), and $\mathbf{R}_{\mathrm{L}}^{-1}$ is readily computed as the inverse of $\mathbf{R}_{\mathrm{L}} \in \mathbb{C}^{2 \times 2}$.

It follows from (13) that all nonzero eigenvectors of $\widetilde{\mathbf{B}}$ will have the structure

$$
\mathbf{q}=\left[\begin{array}{c}
\mathbf{0} \\
\mathbf{q}_{\mathrm{L}}
\end{array}\right]
$$

where $\mathbf{q}_{\mathrm{L}} \in \mathbb{C}^{2}$. The optimal eigenvector $\mathbf{q}^{\star}$ associated with the largest eigenvalue of $\widetilde{\mathbf{B}}$ is thus efficiently computed from the reduced sized 2 dimensional eigenvalue problem

$$
\widetilde{\mathbf{B}}_{\mathrm{L}} \mathbf{q}_{\mathrm{L}}=\lambda_{\max } \mathbf{q}_{\mathrm{L}}
$$

which is easily solved in closed form. The optimal channel vector $\mathbf{h}^{\star}$ and equalizer $\mathbf{d}^{\star}$ are next obtained by first computing the optimal $\mathbf{p}^{\star}$ by back substitution of $\mathbf{R} \mathbf{p}^{\star}=\mathbf{q}^{\star}$.

The alert reader will notice that the optimal channel and equalizer are not unique. From (10), it follows that the SINR is unaffected by scaling and phase rotation of $\mathbf{p}$. To ensure a unique solution, the optimal channel and equalizer are normalized and phase rotated to satisfy

$$
\left[\mathbf{d}^{\star}\right]_{1} \in \mathbb{R}_{+} \quad\left\|\mathbf{d}^{\star} \tilde{\mathbf{Z}}-\mathbf{h}^{\star} \mathbf{X}\right\|^{2}=32,
$$

where the first statement requires the first equalizer tap to be real and positive and the second normalizes the residual noise such that each element has an average power of 1 . The normalization can be computed very efficiently by taking advantage of the previously computed $\mathbf{q}_{\mathrm{L}}$. For any SINR maximizing $\mathbf{p}$ it holds that

$$
\|\mathbf{d} \tilde{Z}-\mathbf{h X}\|^{2}=\mathbf{p}^{*} \mathbf{A p}=\mathbf{q}^{*} \mathbf{q}=\mathbf{q}_{\mathrm{L}}^{*} \mathbf{q}_{\mathrm{L}}
$$

where $\mathbf{q}_{\mathrm{L}}$ is the solution to (15). The normalized optimal $\mathbf{p}^{\star}$ can thus be obtained as

$$
\mathbf{p}^{\star}=\sqrt{32} \frac{\left|p_{1}\right|}{\| \mathbf{q}_{\mathrm{L}}|| p_{1}} \mathbf{p},
$$

where $p_{1}$ denotes the first element of $\mathbf{p}$, which is the SINR maximizing solution associated with $\mathbf{q}_{\mathrm{L}}$.

It should be mentioned that there is an inherent risk of over fitting the equalizer to the particular noise realization. The maximization is therefore (Tikhonov) regularized $[14,15]$ with respect to $\mathbf{d}$, see Algorithm 1 . The 
regularization weight, $\delta$, is chosen to be of the same order as the noise power. In implementation, the weight is obtained as

$$
\delta=10^{-4} \frac{\operatorname{Tr}\left\{\tilde{\mathbf{Z}} \tilde{\mathbf{Z}}^{*}\right\}}{9},
$$

which approximately corresponds to an SNR of $40 \mathrm{~dB}$. The regularization further affects the normalization (18), which, taking regularization into account, becomes,

$$
\mathbf{p}^{\star}=\sqrt{32} \frac{\left|p_{1}\right|}{p_{1} \sqrt{\left\|\mathbf{q}_{\mathrm{L}}\right\|^{2}-\delta\|\mathbf{d}\|^{2}}} \mathbf{p},
$$

where $\mathbf{d}$ is the (unnormalized) equalizer, associated with $\mathbf{p}$. The algorithm is summarized in Algorithm 1.

\subsubsection{Implementation issues}

Above a detailed description of the channel and equalizer estimation was given. It was assumed that transmitted data in the frame $\mathbf{X}$ and the received buffer $\widetilde{\mathbf{Z}}$ was known and was used to form the correlation matrices $S \in \mathbb{C}^{2 \times 2}, \mathbf{Q} \in \mathbb{C}^{9 \times 9}$, and $\mathbf{P} \in \mathbb{C}^{2 \times 9}$, see Algorithm 1 .

However, as more frames are received, it is desirable to exploit the information provided by the previous frames when computing the new channel and equalizer estimates and thereby increase performance. The correlation matrices are therefore updated autoregressively. Let $\{\cdot\}^{(n)}$ denote a matrix associated with frame $n$. The correlation matrices of frame $n$ are thus obtained as

$$
\mathbf{S}^{(n)}=\gamma \mathbf{X}^{(n)}\left(\mathbf{X}^{(n)}\right)^{*}+(1-\gamma) \mathbf{S}^{(n-1)},
$$

and $\mathbf{P}^{(n)}$ as well as $\mathbf{Q}^{(n)}$ are updated similarly. Assuming that the system has been run infinitely long, these autoregressive updates make the channel and equalizer maximize

$$
\frac{\sum_{k=-\infty}^{n} \gamma(1-\gamma)^{n-k}\left\|\mathbf{h} \mathbf{X}^{(k)}\right\|^{2}}{\sum_{k=-\infty}^{n} \gamma(1-\gamma)^{n-k}\left\|\mathbf{d} \widetilde{\mathbf{Z}}^{(k)}-\mathbf{h} \mathbf{X}^{(k)}\right\|^{2}},
$$

where the regularization has been disregarded.

Hence the old frames still contribute to the optimization, but their influence decreases exponentially. The choice of $\gamma$ is a tradeoff of how fast the system should track environmental changes and how well it should estimate the correlation matrices. In the implementation, $\gamma$ is set to 0.75 to allow a quite rapid adaptation. When $\gamma$ is chosen too small, the main cause of error seems to be that the system is unable to adaptively track rotations of the constellation caused by relatively small errors in the carrier offset compensation.

\subsubsection{Choice of equalizer taps}

Another important issue, concerning the implementation of the temporal equalizers that remains to be discussed, is how the taps of the equalizer should be spaced. Note that when there is a complexity constraint on the temporal equalizer, as is the case here, it may not be preferable to let the equalizer taps be uniformly spaced around the expected symbol position. This is because the equalizer should, apart from the current symbol, be provided with as much information as possible about the influencing ISI. When the number of equalizer taps is limited, due to the complexity constraint, it is beneficial to have a nonuniform spacing of the taps.

From Figure 7, it is apparent that a particular symbol interferes primarily with the three symbols transmitted prior and the three symbols transmitted after. The maximum information about these interfering symbols are provided by the 6 samples with relative position $\{ \pm 5, \pm 10, \pm 15\}$ to the sample corresponding to the current symbol. Further, the equalizer is provided with information about the current symbol using the samples with relative position $\{-1,0,1\}$. This gives a protection to small subsample synchronization errors.

To summarize, the 9 taps of the equalizer are chosen to operate on the samples with relative sample position

$$
\{0, \pm 1, \pm 5, \pm 10, \pm 15\}
$$

to the current symbol.

\subsection{Spatial equalization}

As stated in Section 3.3 the spatial channel $\mathbf{H}$ can be decoupled into two noninterfering spatial streams of unit gain by preprocessing by $\mathbf{V}$ and postprocessing by $\boldsymbol{\Sigma}^{-1} \mathbf{U}^{*}$. In practice, there is a feedback delay, and when the preprocessing matrix $\mathbf{V}$ is applied, the channel matrix may have changed slightly. Let $\mathbf{H}$ be the current channel matrix and let $\hat{\mathbf{V}}$ be the precoder matrix which is currently being used by the transmitter. Again, note that due to the feedback delay, $\hat{\mathbf{V}}$ may not necessarily be equal to the left singular vectors of $\mathbf{H}$. However, under the assumption of slow fading, it is likely that $\hat{\mathbf{V}}$ is close to $\mathbf{V}$.

The postprocessing matrix used in the actual implementation is given by

$$
\mathbf{G} \triangleq \hat{\mathbf{V}}^{*}\left(\mathbf{H}^{*} \mathbf{H}+\mathbf{I}\right)^{-1} \mathbf{H}^{*}
$$

where the equalizer being scaled to give unit noise variance is used, see Section 5.1. This is the linear MMSE spatial equalizer for the channel given by $\mathbf{H} \hat{\mathbf{V}}$; that is, the channel from $\mathbf{c}$ in Figure 10 to $\mathbf{y}$ in Figure 11. In the case where $\hat{\mathbf{V}}=\mathbf{V}$, for $\mathbf{H}=\mathbf{U} \mathbf{\Sigma} \mathbf{V}^{*}$, the processing matrix $\mathbf{G}$ is given by

$$
\mathrm{G}=\mathrm{DU}^{*},
$$

where

$$
\mathbf{D}=\operatorname{diag}\left(\frac{\sigma_{i}}{\sigma_{i}^{2}+1}\right)=\operatorname{diag}\left(\frac{1}{1+\sigma_{i}^{-2}}\right) \Sigma^{-1} .
$$

Thus, given that the assumption of a constant $\mathbf{H}$ is true, this equalizer is equivalent to $\Sigma^{-1} \mathbf{U}^{*}$ except for a small bias which can be accounted for prior to detection. However, the matrix of (24) tends to be more robust to deviation of $\hat{\mathbf{V}}$ from $\mathbf{V}$ and is for this reason used instead of $\Sigma^{-1} \mathbf{U}^{*}$. 


\subsection{Feedback}

After decoding a 32 symbol frame a new precoder matrix $\mathbf{V}$ is computed using the SVD of the current channel estimate $\mathbf{H}$, that is,

$$
\mathbf{H}=\mathbf{U} \boldsymbol{\Sigma} \mathbf{V}^{*} \text {. }
$$

The precoder must be fed back to the transmitter together with the requested data rates for the two spatial data streams. In the current implantation, a 32 bit word may be fed back during each frame. At first, this may seem limited to describe the 2 by 2 complex matrix $\mathbf{V}$. However, as $\mathbf{V}$ is structured the feedback capacity is more than enough.

First, note that the SVD is only unique up to a complex rotation of the corresponding columns of $\mathbf{U}$ and $\mathbf{V}$. Thus, without loss of generality, the first row of $\mathbf{V}$ may be assumed real valued. This removes 2 degrees of freedom. Further, it is known that the columns of $\mathbf{V}$ are orthogonal and have unit norm; this removes an additional 4 degrees of freedom. Therefore, there is only 2 degrees of freedom left in V. A suitable parametrization of $\mathbf{V}$ is given by

$$
\mathbf{V}(s, t)=\left[\begin{array}{cc}
\cos (s) & \sin (s) \\
-\sin (s) e^{\mathrm{jt}} & \cos (s) e^{\mathrm{jt}}
\end{array}\right] \text {, }
$$

where $0 \leq s \leq \pi / 2$ and $0 \leq t \leq 2 \pi$. An additional benefit of such a parametrization is that the free parameters, $s$ and $t$, belong to a finite range and are therefore easily quantized to the desired precisions. Similar parameterizations and extensions to matrices larger than 2 by 2 are treated in [16].

A total of 6 bits are required for feedback of data rates leaving 26 bits for feedback of $s$ and $t$. In the current implementation, 12 bits are used for $s$ and 14 bits for $t$. This yields an absolute precision of approximately $4 \times 10^{-4}$, which is more than required.

\subsection{Cause of bit error}

When designing a system with adaptive rate, it is crucial for the system to have accurate information of the current SINRs of the two spatial streams. The SINR of each spatial stream is adaptively measured at the receiver using decision feedback of the decoded data. Using the estimated SINRs, the highest supported transmission rate is chosen; as discussed in Appendix A.

There are in this procedure two effects that must be taken into consideration. Firstly, there will inevitably be some estimation errors in the SINR estimates. Secondly, due to the feedback delay, the SINR estimates will be inaccurate when the channel experiences fast fading. The first issue may be resolved by averaging over a larger number of samples. This will however affect the ability to track the channel during fast fading. To combat these difficulties rates are chosen with a margin in the SINR required to maintain a target BER over a nonfading channel. The size of this fade margin should reflect the amount of fading which may occur within the time span of the feedback delay. Naturally, by increasing this margin the system becomes more robust to changes in the environment. However, a large margin will decrease the overall throughput.

For a reasonable margin, the errors will come in bursts which occur when the system is unable to track the channel. This effect is noted in the measurements of Section 7. In a full scale communication system, it may be desirable to allow for a fairly large probability of an error burst and use retransmissions to protect against such events. Herein, as we focus on physical layer implementation, no such retransmissions are made. Instead, BER is kept low by using a larger margin and thereby reducing the data rate slightly.

\section{THE MEMORYLESS AWGN MODEL}

Temporal equalization was implemented as outlined in Section 4. However, the question of how accurately the resulting channel is described by (1) was left unaddressed. Also, there is a question of how much is lost in terms of performance by adopting the multiple-layer approach as opposed to a joint solution. In this section both issues are addressed.

For evaluation purposes it is interesting to estimate the SNR of the wireless channel without the contribution of hardware induced ISI and nonlinearities and compare it with (1). This estimation is accomplished by feeding the input $x_{1}$ and $x_{2}$ of Figure 5, with (complex-valued) sinusoidal signals. The sinusoids are positioned in the center of the frequency band. The power and phase-rotation of these signals were measured at the receiver. Further, the noise in each receiver chain was measured during a period of no transmission. This yields a model similar to that of (1), that is,

$$
\tilde{\mathbf{y}}(n)=\tilde{\mathbf{H}} \tilde{\mathbf{x}}(n)+\widetilde{\mathbf{w}}(n) .
$$

Note that the channel obtained in this fashion is relatively unsensitive to effects of frequency offset, synchronization, and ISI. It may therefore be used as an indicator of what is achievable in terms of performance, were it not for these degrading effects. It should also be noted that the procedure of obtaining $\tilde{\mathbf{H}}$ is not considered part of the communication system but is included for evaluation purposes only. This quantity is of little use in a real system and obtaining it reduce the overall data rate.

Ideal SNRs of the two spatial streams are obtained as

$$
\mathrm{SNR}_{i}^{\mathrm{S}} \triangleq \frac{\sigma_{i}^{2}(\tilde{\mathbf{H}}) \tilde{E}_{s}}{\tilde{N}_{0}}
$$

where $\sigma_{i}(\cdot)$ denotes the $i$ th singular value and where $\widetilde{E}_{s}, \tilde{N}_{0}$ are the power of $\widetilde{\mathbf{x}}(n)$ and $\tilde{\mathbf{w}}(n)$, respectively. The superscript " $S$ " indicates that the SNR is that of a spatial stream in order to avoid confusion with the SNRs and SINRs of the receiver chains discussed in Section 2.

An estimate of the actual SINR of each spatial stream at the input of the TCM decoder is estimated as

$$
\operatorname{SINR}_{i}^{S} \triangleq \frac{\sum_{n}\left|c_{i}(n)\right|^{2}}{\sum_{n}\left|\hat{c}_{i}(n)-c_{i}(n)\right|^{2}},
$$

where $c_{i}(n)$ and $\hat{c}_{i}(n)$ are given in Figures 5 and 6 , and $\widehat{c}_{i}$ should be well modelled by (2). Since the true data 


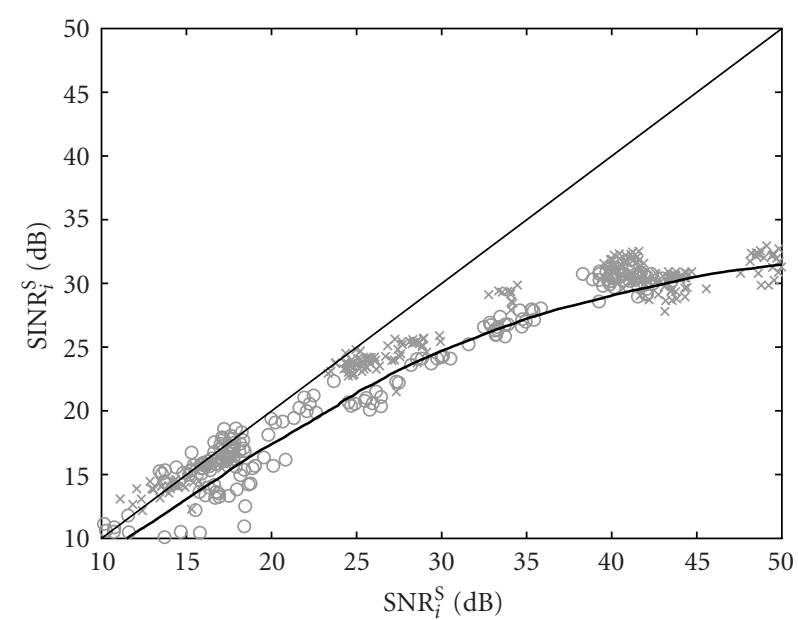

FIgURE 12: $\operatorname{SINR}_{i}^{S}$ as a function of $\operatorname{SNR}_{i}^{S}$. The crosses indicates a measurement from the first (stronger) spatial stream and the circles the second.

stream $c_{i}(n)$ is not available at the receiver, except during training, it is approximated using decision feedback of the decoded bit stream.

A comparison of $\mathrm{SNR}_{i}^{\mathrm{S}}$ and $\mathrm{SINR}_{i}^{\mathrm{S}}$, computed from measurements, indicate how much is lost in terms of performance due to effects such as temporal ISI. Such a comparison is undertaken in what follows.

\subsection{Signal-to-interference ratio}

In Figure 12 the $\operatorname{SINR}_{i}^{S}$ is plotted as a function of $\mathrm{SNR}_{i}^{S}$. Different scenarios were created by physically moving the transmitter and by using attenuators at the transmitter antennas. As can be seen from the figure, there is a loss of approximately $2 \mathrm{~dB}$ from SNR to SINR in the low to mid SNR region. In this region, the SINR is dominated by the noise component. However, as the SNR of the system grows large, the effect of self interference becomes increasingly notable and the SINR curve flattens out. This indicates that there is a substantial amount of ISI which the temporal equalizer is unable to suppress. It should however be mentioned that without the temporal equalizer, SINRs of at most $17 \mathrm{~dB}$ are achievable on the current hardware; assuming sub-sample synchronization. Thus, the temporal equalizer substantially increase the range of SNR for which an increase in transmission power may yield an increase in system throughput.

\subsection{Noise statistics}

Figure 13 illustrates the statistical properties of the noise in (1), or more precisely, the residual obtained by the optimization in (9). The data was obtained when the system was operating at a point where $\mathrm{SNR}_{1}^{S} \approx 25 \mathrm{~dB}$, that is, where the curves break apart in Figure 12. The figure only shows data obtained from the first receive antenna chain, that is, the noise sequence $w_{1}(n)$ and the signal given by $s_{1}(n) \triangleq \mathbf{h}_{1} \mathbf{x}(n)$,

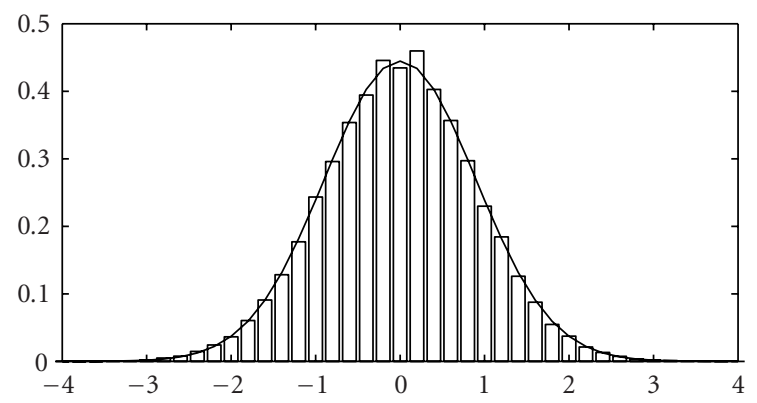

(a)

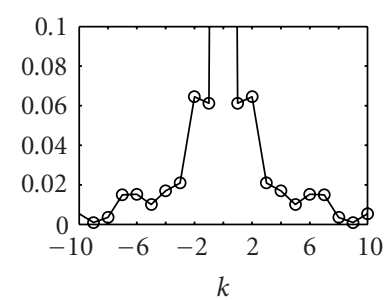

(b)

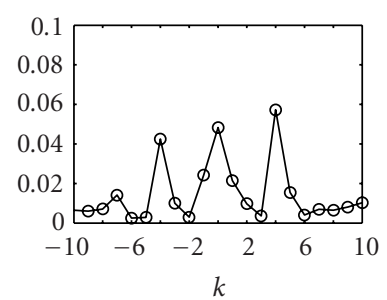

(c)
FIgURE 13: Statistical properties of the residual noise in (9). The noise is normalized to unit variance. (a)Noise histogram. (b)Noise autocorrelation, $\rho_{w}(k)$. (c)Cross-correlation, $\rho_{w s}(k)$.

where $w_{1}(n)$ and $\mathbf{h}_{1}$ are the first component and row of $\mathbf{w}(n)$ and $\mathbf{H}$, respectively, which are given in (1). The data for the second chain shows similar characteristics and is therefore not included.

Figure 13(a) shows a measured histogram of $w_{1}(n)$, including both real and imaginary components. As can be seen from the figure, the Gaussian noise assumption often made for (1) is well satisfied. Figures 13(b) and 13(c) show the autocorrelation of $w_{1}(n), \rho_{w}(k)$, and the cross-correlation between $w_{1}(n)$ and $s_{1}(n), \rho_{w s}(k)$. The value of $\rho_{w}(k)$ and $\rho_{w s}(k)$ are obtained as

$$
\begin{aligned}
\rho_{w}(k) & \triangleq \frac{\sum_{n} w_{1}^{*}(n) w_{i}(n+k)}{\sum_{n}\left|w_{1}(n)\right|^{2}}, \\
\rho_{w s}(k) & \triangleq \frac{\sum_{n} w_{1}^{*}(n) s_{1}(n+k)}{\sqrt{\sum_{n}\left|w_{1}(n)\right|^{2}} \sqrt{\sum_{n}\left|s_{1}(n)\right|^{2}}},
\end{aligned}
$$

where $s_{1}(n)=\mathbf{h}_{1} \mathbf{x}(n)$. Note that the normalization is such that $\left|\rho_{w}(k)\right| \leq 1$ and $\left|\rho_{w s}(k)\right| \leq 1$. Since the correlation between noise samples separated in time, as well as between the signal and noise, is at most 0.06 , the memoryless assumption often made for (1) is highly accurate. It should however be mentioned that as the SNR of the system is increased, by moving operations into the region where ISI is poorly suppressed by the temporal equalizers, the correlation will increase.

It is interesting to note the peaks for a sample separation of $k= \pm 4$ in Figure 13(c). These peaks are the result of the equalizer which only operates on the three symbols prior and after the current symbol, and is thus unable to suppress the correlation to symbols more than 3 symbol samples away. 
By the data presented in Figure 13, it can be concluded that the discrete-time, memoryless AWGN model given in (1) is accurate up to $20-25 \mathrm{~dB}$ SNR for the current system. Further, the performance loss due to temporal equalization prior to spatial processing, as opposed to a joint design, is small for these SNRs. However, at high SNR, the effects of hardware induced ISI are more severe, rendering such assumptions questionable.

\section{SYSTEM PERFORMANCE}

In this section, system performance is evaluated in terms of throughput for several different indoor, non-line-of-sight scenarios. These were, as the measurement data in Section 6, created by moving the transmitter and attenuating the transmitted signals. The receiver was located in an office while the transmitter was located in an adjacent corridor. The distance between transmitter and receiver varied from 3 to 20 meters.

To assess the benefit of employing a MIMO system as opposed to a SISO system, a comparable single transmit and receive antenna system was designed and implemented. The system uses the same type of temporal equalizer and coding as the MIMO system. In order to enable a relevant comparison between the systems, the same hardware is used; that is, the 1 by 1 system is implemented using one receive and one transmit antenna of the multiple-antenna system. During operations, the MIMO and SISO systems are run in 0.2 second (duration of a super frame) interleaved time slots. This enables a comparison of the two systems under similar channel characteristics.

The output power was adjusted such that the total transmitted energy is the same for both systems. Additionally, to ensure fair comparison, all possible combinations of transmit and receive antenna pairs were used in the SISO system for each transmitter location and setting. This reduces the risk of only creating scenarios where the path gain between the SISO antennas is lower or higher than those experienced in the MIMO system.

The measurement data was collected during 24 different scenarios each consisting of 10 super frames. Thus, a total of 240 transmission bursts (super frames) were recorded and a total of $4.2 \mathrm{Mb}$ (information bits) were transmitted. The reported throughput figures are the total number of bits transmitted during one such super frame divided by the duration of the frame. The average BER, averaged over the total 240 super frames, was $4.3 \times 10^{-5}$. However, the errors tend to occur in bursts, as discussed in Section 5.4, and all bit errors are located in only 3 different super frames.

In what follows, the throughput achievable on each spatial channel is reported. This is followed by comparisons between the single-and multiple-antenna systems.

\subsection{Throughput of the spatial channels}

Figure 14 shows the system throughput in bits per symbol for the two spatial streams. A throughput estimate provided by the gap approximation is included as a reference. The gap approximation provides an accurate estimate of the rate

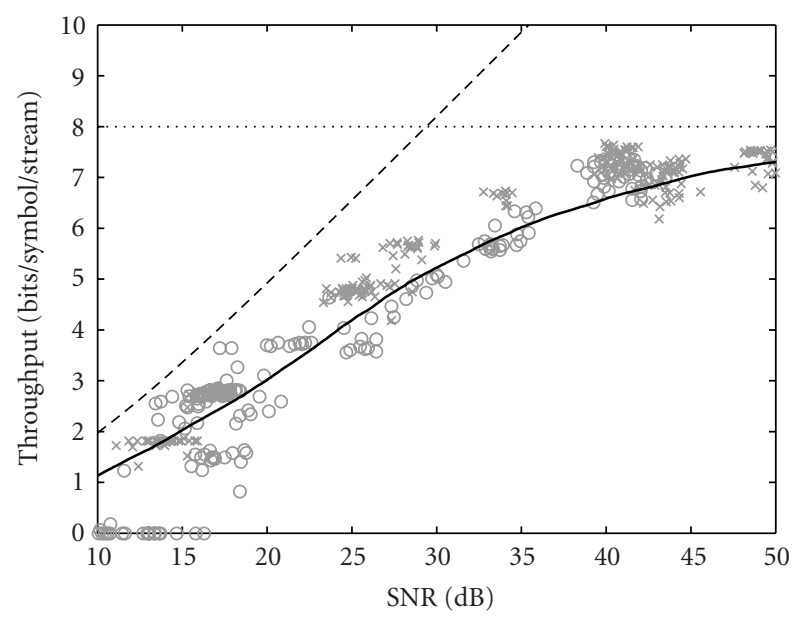

Figure 14: Average throughput in bits per symbol and spatial stream for given values of $\mathrm{SNR}_{i}^{S}$ defined in (30). The crosses indicates a measurement from the first (stronger) spatial stream and the circles the second. The dotted line shows the limit of 8 bits per symbol and stream given by the maximum constellation size. The dashed line shows an estimate of the achievable rate over a flat AWGN channel given by the gap approximation for the corresponding SNR. The solid line is simply a smooth line fit to the data.

(in bits per channel use) over a nonfading AWGN channel, given SNR, probability of error, and code [17]. The rate is, according to this approximation, given by

$$
b=\log _{2}\left(1+\frac{\mathrm{SNR}}{\Gamma}\right)
$$

where the gap $\Gamma$ is a measure of how far from channel capacity the system is operating. Herein, we use a gap of $\Gamma=$ $7.0 \mathrm{~dB}-4.7 \mathrm{~dB}=2.3 \mathrm{~dB}$ which correspond to the gap for uncoded BER of $5 \times 10^{-5}$ minus a $4.7 \mathrm{~dB}$ coding gain corresponding to the effective gain of the TCM code. More information about computing the gap is given in [17].

As could have been predicted from the results in Section 6, the system operates closer to the limit given by the approximation in the range of SNR where the ISI is relatively low. The throughput will also saturate when the SNR increases. When choosing the constellation size, an SINR margin of $2 \mathrm{~dB}$ to the SNR required for a BER of $10^{-6}$ (on the AWGN channel) was used. The reason for this, as discussed in Section 5.4, is the problem of accurately tracking the instantaneous SINR of the channel. By increasing the margin, the system becomes more robust to rapid changes in SINR. However, this comes at the cost of a reduced overall throughput.

The solid line in Figure 14 is a smoothed and averaged throughput curve. Note, however, the staircase nature of the measured data due to the integer constrained constellation sizes. The throughput will thus only be close to the gap approximation when operating close to the constellation transition points. 


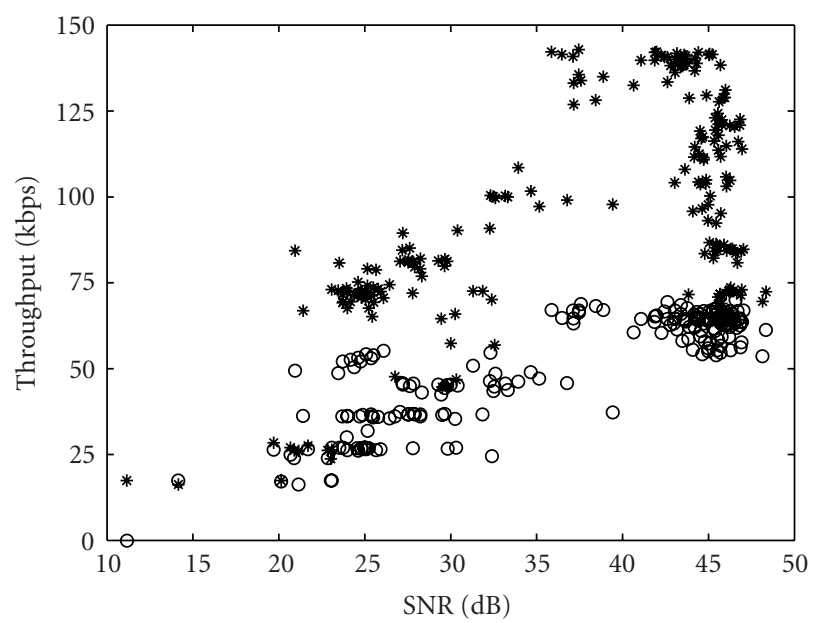

FIgURE 15: Throughput of the MIMO and SISO systems given the instantaneous SNR of the SISO channel. The crosses indicate measurements from the $2 \times 2$ system and the circles the $1 \times 1$ system.

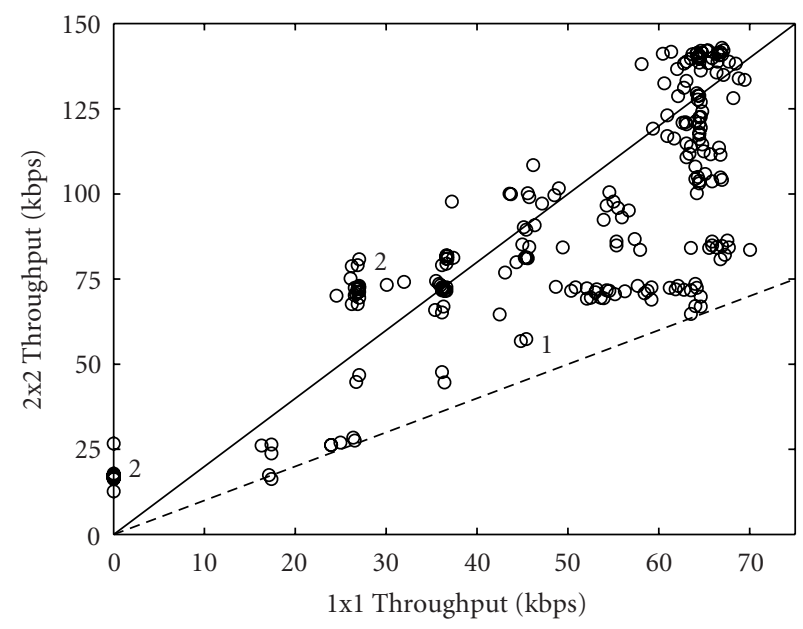

FIgUre 16: Comparison of SISO and MIMO throughput for two consecutive time slots. The dashed line indicates equal throughput and the solid line when the MIMO system throughput is twice that of the SISO system. The number 2 indicate events where the MIMO system provides a substantial gain over the SISO system. The number 1 indicate equal performance.

\subsection{MIMO versus SISO}

Figures 15-17 show the performance of the multipleantenna system as compared to the single-antenna system. In Figure 15, the average throughput of the two systems is shown as a function of the instantaneous SNR of the singleantenna system; that is,

$$
\mathrm{SNR}_{1 \times 1} \triangleq \frac{|\tilde{h}|^{2} E_{s}}{N_{0}}
$$

where $\tilde{h}$ is the complex baseband equivalent gain of the single-antenna channel obtained by transmission of sinusoidal signals as outlined in Section 6. $E_{s}$ is the constellation

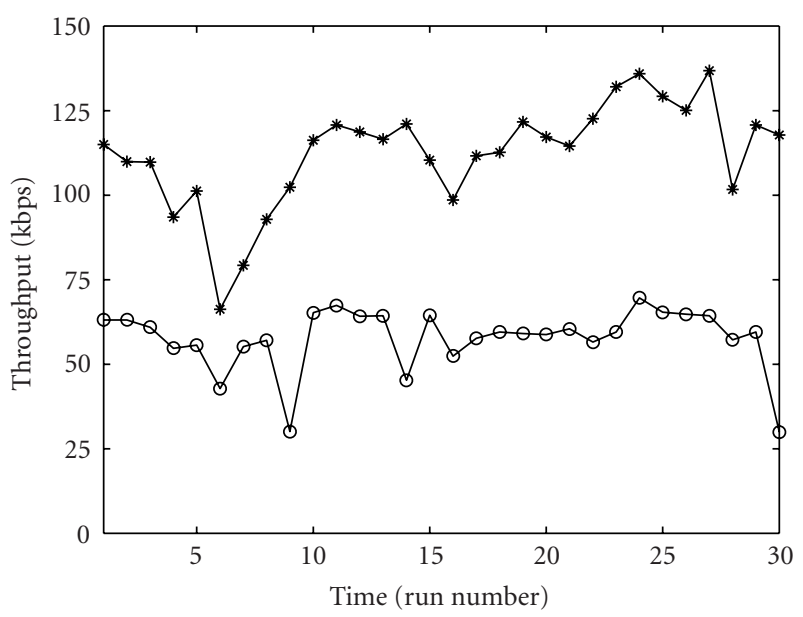

FIGURE 17: Throughput of MIMO and SISO systems as a function of time for a non-line-of-sight scenario where the transmitter is moving slowly down a corridor towards the receiver. The MIMO system throughput indicated by crosses and the SISO system throughput by circles.

energy of the single-antenna system. Note again that the throughput reported is information bits per unit time averaged over one super frame.

In Figure 16 throughput pairs are shown. On the vertical axis the throughput of the 2 by 2 system is shown while the throughput of the 1 by 1 system is shown on the horizontal axis. The throughput pairs were obtained by measuring throughput on the two systems during two consecutive super frames. Since the transmitter and receiver were stationary during these measurements, the channels seen by the two implementations may be considered comparable. Two important events, clearly seen in the figure, are interesting to illuminate. Firstly, it may be the case that $\tilde{h}$ is small even though the MIMO channel $\mathbf{H}$ is large. This corresponds to the case when the path gain of the single-antenna system is in a deep fade, but several other gains of the multiple-antenna system are strong. In this case, the multiple-antenna system may be able to reliably transmit data while single-antenna system is unable to do so. Events that are likely caused by this are indicated by the number 2 in Figure 16. Secondly, it could happen that the channel matrix given by $\mathbf{H}$ is close to rank one implying that only one of the spatial streams are able to sustain reliable transmission. During such event, the throughput of the two systems are likely to be similar. These events are characterized by throughput pairs which are close to the dashed line in Figure 16 and are marked by the number 1 .

As can be concluded from the above, under comparable conditions, the MIMO system achieves roughly twice the throughput of the SISO system. For some realizations, the factor is even larger. In Figure 17, the throughput of the two systems are shown as a function of time during 30 time slots while the transmitter was slowly moving towards the receiver. This data further strengthens the conclusion that, under otherwise comparable conditions, approximately twice the data 
rate can be expected when using two transmit as well as receive antennas instead of a single-antenna system.

\section{CONCLUSIONS}

A 2 by 2 MIMO system utilizing spatial multiplexing was implemented and evaluated. It was shown that it is possible to operate close to the theoretical limits using a layered design, which greatly simplifies the design of the coding, spatial processing, and temporal processing.

It was further shown how off-the-shelves radio equipment can be used in high-performance systems; by mitigating hardware induced ISI using advanced signal processing to equalize the received signal. An optimal linear joint equalizer and channel estimator was derived, and a low complexity implementation made it possible to realize. The proposed algorithm differs from other proposed techniques in that no assumptions on the transmitted data are necessary.

The equalized signal was shown to be well approximated by the memoryless linear MIMO channel, with AWGN. This simplifies the design of the spatial processing, in that the system is well approximated by the memoryless discrete time channel model often used in the literature.

Spatial multiplexing was implemented, which in combination with adaptive TMC (coding) enabled a total throughput of up to $150 \mathrm{kbps}$ over a $9.6 \mathrm{kHz}$ channel. This corresponds to a spectral efficiency of more than $15 \mathrm{bps} / \mathrm{Hz}$, with BER less than $5 \times 10^{-5}$.

The 2 by 2 MIMO system was compared with the corresponding 1 by 1 system utilizing the same temporal processing and coding. The MIMO system had a performance gain of more than twice the throughput of the SISO system.

\section{APPENDIX}

\section{A. TCM}

The code used within the spatial layer is a rate $2 / 3$ 64-state TCM code $[10,11]$ with generator polynomials

$$
G(D) \triangleq\left[\begin{array}{ccc}
1 & D^{3}+D^{2} & 0 \\
D^{3}+1 & D^{3}+D^{2}+D+1 & D^{2}
\end{array}\right] .
$$

This code has a coding gain of $5.44 \mathrm{~dB}$ and an effective gain (when compensating for an increase in number of nearest neighbors) of $4.72 \mathrm{~dB}$ according to [17, Table 10.8], where the corresponding parity matrix (in octals) is 060004143.

The encoder is forced to the zeroth state at the end of each frame, consisting of 32 symbols. This allows the decoder to decode each frame separately, which simplifies the implementation.

The rate of transmission for each spatial stream is adapted by changing the size of the constellation from 8 PSK to 512 cross QAM in steps of one bit. The available modes are listed in Table 3 along with constellation and data rate in kbps. Also listed is the minimum required SNR to achieve a BER lower than $10^{-6}$, over an AWGN channel.

An uncoded BPSK mode is included for the event when no other mode is supported on a spatial channel. This mode
TABLE 3: Data rates of different transmission modes.

\begin{tabular}{clccc}
\hline Mode & Constellation & Bits per frame & Data rate & Min. SNR \\
\hline 0 & BPSK & - & - & - \\
1 & 8 PSK & 58 bits & $17.4 \mathrm{kbps}$ & $9.5 \mathrm{~dB}$ \\
2 & 16 QAM & $90 \mathrm{bits}$ & $27.0 \mathrm{kbps}$ & $13.1 \mathrm{~dB}$ \\
3 & 32 cross QAM & $122 \mathrm{bits}$ & $36.6 \mathrm{kbps}$ & $16.3 \mathrm{~dB}$ \\
4 & 64 QAM & $154 \mathrm{bits}$ & $46.2 \mathrm{kbps}$ & $19.5 \mathrm{~dB}$ \\
5 & 128 cross QAM & $186 \mathrm{bits}$ & $55.8 \mathrm{kbps}$ & $22.5 \mathrm{~dB}$ \\
6 & 256 QAM & 218 bits & $65.4 \mathrm{kbps}$ & $25.7 \mathrm{~dB}$ \\
7 & 512 cross QAM & 250 bits & $75.0 \mathrm{kbps}$ & $28.6 \mathrm{~dB}$ \\
\hline
\end{tabular}

is, however, only used for transmitting training data in the event that the channel becomes usable during the super frame.

Decoding of the TCM is done using the Viterbi algorithm, where the path metric is the distance to the closest constellation coset. Each of the transmission modes from 16 QAM and up include uncoded, systematic bits which are protected by the separation of points within a coset.

\section{ACKNOWLEDGMENT}

The authors would like to thank Dr. Sarah Kate Wilson for suggesting, explaining, and answering numerous questions regarding TCM coding.

\section{REFERENCES}

[1] G. J. Foschini and M J. Gans, "On limits of wireless communications in a fading environmen when using multiple antennas," Wireless Personal Communications, vol. 6, no. 3, pp. 311335, 1998.

[2] I. E. Telatar, "Capacity of multi-antenna Gaussian channels," European Transactions on Telecommunications, vol. 10, no. 6, pp. 585-595, 1999.

[3] L. Zheng and D. N. C. Tse, "Diversity and multiplexing: a fundamental tradeoff in multiple-antenna channels," IEEE Transactions on Information Theory, vol. 49, no. 5, pp. 1073-1096, 2003.

[4] G. G. Raleigh and J. M. Cioffi, "Spatio-temporal coding for wireless communication," IEEE Transactions on Communications, vol. 46, no. 3, pp. 357-366, 1998.

[5] G. J. Foschini, "Layered space-time architecture for wireless communication in a fading environment when using multielement antennas," Tech. Rep., Lucent Technologies, Murray Hill, NJ, USA, 1996.

[6] P. W. Wolniansky, C. J. Foschini, G. D. Golden, and R. A. Valenzuela, "V-BLAST: an architecture for realizing very high data rates over the rich-scattering wireless channel," in Proceedings of URSI International Symposium on Signals, Systems, and Electronics (ISSSE '98), pp. 295-300, Pisa, Italy, September-October 1998.

[7] T. Horseman, J. Webber, M. K. Abdul-Aziz, et al., "A software and hardware evaluation of revolutionary turbo MIMO OFDM schemes for $5 \mathrm{GHz}$ WLANs," in Proceedings of 57th IEEE Semiannual Vehicular Technology Conference (VTC '03), vol. 4, pp. 2788-2792, Jeju, Korea, April 2003.

[8] H. Sampath, S. Talwar, J. Tellado, V. Erceg, and A. Paulraj, "A fourth-generation MIMO-OFDM broadband wireless system: 
design, performance, and field trial results," IEEE Communications Magazine, vol. 40, no. 9, pp. 143-149, 2002.

[9] P. Zetterberg, "WIreless DEvelopment LABoratory (WIDELAB) equipment base," Tech. Rep. S3-SB-0316, Department of Signals, Sensors and Systems, Royal Institute of Technology (KTH), Stockholm, Sweden, August 2003.

[10] G. Ungerboeck, "Trellis-coded modulation with redundant signal sets Part I: Introduction," IEEE Communications Magazine, vol. 25, no. 2, pp. 5-11, 1987.

[11] G. Ungerboeck, "Trellis-coded modulation with redundant signal sets Part II: State of the art," IEEE Communications Magazine, vol. 25, no. 2, pp. 12-21, 1987.

[12] P. H. Moose, "A technique for orthogonal frequency division multiplexing frequency offset correction," IEEE Transactions on Communications, vol. 42, no. 10, pp. 2908-2914, 1994.

[13] S. Fanfoni, D. Giancola, U. Girola, S. Parolari, A. Picciriello, and U. Spagnolini, "Space-time processing for co-channel interference rejection and channel estimation in GSM/DCS systems," in Proceedings of URSI International Symposium on Signals, Systems, and Electronics (ISSSE '98), pp. 152-155, Pisa, Italy, September-October 1998.

[14] Å Björck, Numerical Methods for Least Squares Problems, SIAM, Philadelphia, Pa, USA, 1996.

[15] A. Neumaier, "Solving ill-conditioned and singular linear systems: a tutorial on regularization," SIAM Review, vol. 40, no. 3 , pp. 636-666, 1998.

[16] D. Agrawal, T. J. Richardson, and R. Urbanke, "Multipleantenna signal constellations for fading channels," IEEE Transactions on Information Theory, vol. 47, no. 9, pp. 2618-2626, 2001.

[17] J. Cioffi, EE379 Class Reader, Stanford University, Stanford, Calif, USA, 1998.

David Samuelsson was born in Stockholm, Sweden in 1977. He received the M.S. degree (with highest honors) in 2003 from the Royal Institute of Technology (KTH), Stockholm, Sweden. In 2001-2002 he pursued M.S. level studies at Stanford University, Stanford, California. He is now working towards the Ph.D. degree in telecommunications in the signal processing group at the Royal Institute of Technology, founded

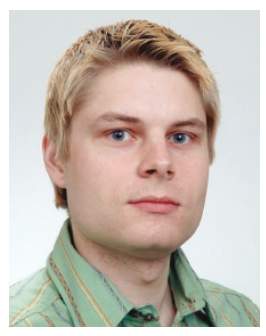
by a grant "Honor Graduate Student Position," from the dean office at the same university. His research interests include wireless communication, beamforming, and user scheduling.

Joakim Jaldén was born in Gävle, Sweden on May 16, 1976. He received the M.S. degree in electrical engineering from the Royal Institute of Technology (KTH), Stockholm, Sweden in 2002. Between September 2000 and May 2002, he studied at Stanford University, California, USA, where he also conducted the research for his M.S. thesis. At present, he is working towards a Ph.D. in telecommunications at the Department of

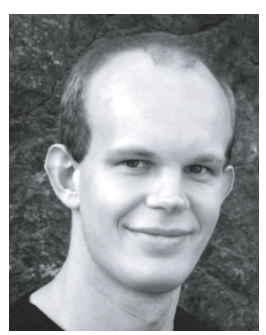
Signal, Sensors \& Systems at the Royal Institute of Technology. The position is funded by a grant, "Honor Graduate Student Position," from the dean office at the Royal Institute of Technology. His research interests include receiver design for MIMO systems and performance and complexity analysis of detection algorithms.
Per Zetterberg was born in Uppsala, Sweden, in 1968. He received the M.S. degree in electrical engineering from Luleå University, Luleå, Sweden in 1993. In 1997, he received the Ph.D. degree in electrical engineering from the Royal Institute of Technology (KTH). Then he joined Radio Design $\mathrm{AB}$ were he worked with smart antenna research and implementation. From 2002 he has rejoined Royal Institute of Technology

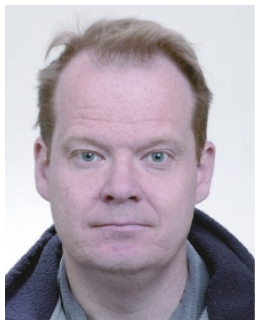
on full time working with radio propagation and testbeds. During 1995-1996, he was a Visiting Researcher at the Center for Personal communication (CPK), Aalborg University, Denmark. Zetterberg's research interests are in the areas of implementation issues and propagation for smart antenna systems.

Björn Ottersten was born in Stockholm, Sweden, 1961. He received the M.S. degree in electrical engineering and applied physics from Linköping University, Linköping, Sweden in 1986. In 1989 he received the Ph.D. degree in electrical engineering from Stanford University, Stanford, California. Dr. Ottersten has held research positions at the Department of Electrical Engineering, Linköping University;

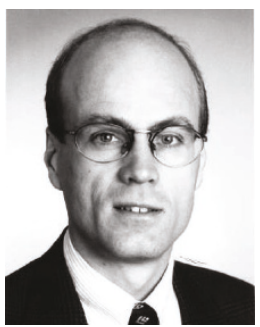
the Information Systems Laboratory, Stanford University; and the Katholieke Universiteit Leuven, Leuven. During 96/97 Dr. Ottersten was Director of Research at ArrayComm Inc, San Jose, California. He has authored papers that received the Signal Processing Society Paper Award in 1993 and 2001. In 1991, he was appointed Professor of Signal Processing at the Royal Institute of Technology (KTH), Stockholm, and he is currently the Dean of the School of Electrical Engineering at KTH. Dr. Ottersten is also a Visiting Professor at the University of Luxembourg. Dr. Ottersten has served as an Associate Editor for the IEEE Transactions on Signal Processing and a Member of the editorial board of EURASIP Journal of Applied Signal Processing. He is currently the Co-Editor in Chief of EURASIP Signal Processing Journal and a Member of the editorial board of the IEEE Signal Processing Magazine. Dr. Ottersten is a Fellow of the IEEE. His research interests include wireless communications, stochastic signal processing, sensor array processing, and time series analysis. 